\title{
Acquisition of Direct-Sequence Ultra-Wideband Signals
}

\author{
Iyappan Ramachandran, Sumit Roy* \\ Department of Electrical Engineering \\ University of Washington \\ Seattle, WA 98105 \\ Email: \{iyappan, roy $\}$ ee.washington.edu \\ ${ }^{*}$ Contact author: Tel/FAX: (206) 221-5261/543-3842
}

\begin{abstract}
Very high rate packet data systems such as those based on ultra-wideband (UWB) signaling face an increasingly important challenge - achieving fast timing acquisition and synchronization (which must be done typically on an individual packet basis) to minimize preamble overhead and optimize (packet) throughput. Impulse based UWB modulation schemes use short (nano-second) time-duration pulses that are appropriately shaped - the resulting high resolution in time implies that the acquisition algorithm must employ sub-pulse duration steps, thereby leading to a large search space if a serial timing acquisition approach is used. Moreover, owing to the strict average transmit power limitations on UWB transmissions resulting from the Part 15 limits imposed by the FCC, a large number of pulses need to be integrated for reliable acquisition decisions, which consequently leads to large mean acquisition times (MAT). In this paper, we investigate the performance of the conventional serial search and random search schemes applied to the acquisition of UWB signals in multipath environments. It is shown that over typical UWB multipath channels, a random search scheme may yield lower MAT than serial search.
\end{abstract}

\section{INTRODUCTION}

Ultra-Wideband (UWB) modulation [1], [2] is the basis for wireless personal area networks (WPAN) within IEEE 802.15.3a standards group intended for use in the $3-10 \mathrm{GHz}$ band on an unlicensed basis in U. S. subject to the Part 15 rules that specify a maximum transmit power spectral density of $-41.3 \mathrm{dBm} / \mathrm{MHz}$. Despite the strict limits on average transmit power, UWB schemes can achieve very high aggregate data rates over short distances due to the very large bandwidths employed [3]. In a multipath dominated environment, such large transmission bandwidths allow for fine time resolution of multipath arrivals, which implies reduced fading per resolved path [4] compared to narrow(er) bandwidth modulation.

Historically, impulse (or narrow pulse) modulation has been used to generate such large transmission bandwidths (order of $\mathrm{GHz}$ ) along with careful shaping of the modulating pulse shape. While several other UWB modulation approaches have been presented in the literature [5], [6], [7], in this work we focus exclusively on a Direct Sequence UWB (DS-UWB) scheme which in principle is very similar to a standard DSSS modulation; this is embodied in a proposal before the 15.3a standard body [8]. In contrast to the raised cosine shaped pulses transmitted at 1.368 Gcps used in [8], we use a baseband pulse shaped as the $2^{\text {nd }}$ derivative of a Gaussian pulse transmitted at 1 Gcps to achieve the necessary spectral control. Our pulse duration is determined so that there is negligible inter-chip-interference at this chip rate. Typically, UWB systems use short codes of length 16 or 32 to achieve code division multiple access (one period of which constitutes a data symbol) resulting in symbol rates of 62 or 31 Msps.

Such narrow pulse widths imply that acquisition algorithms should employ correspondingly narrow step sizes which could lead to potentially large search spaces. The low transmission power spectral density further indicates a need to integrate several pulses for reliable decisions, which also increases the mean acquisition times. However, the fine time resolution results in a (very) large number of significant resolved multipath components, which indicates a potential for it's judicious exploitation to reduce the acquisition times. In summary, design of acquisition schemes for UWB systems have significant differences from those for conventional wideband systems and accordingly require new analytical approaches.

Initial development and analysis of acquisition schemes ([9] and [10], for example) were made with the assumption that there exists only one resolvable path and consequently only one $H_{1}$ cell (in-phase cell). The analysis of the effect of the presence of multiple $H_{1}$ cells on the performance of a serial correlator has been investigated in [11] and [12], where it has been assumed that the number of in-phase cells $\left(H_{1}\right.$ cells) is negligibly smaller than the total number of cells that an acquisition receiver has to test, which is characteristic of wideband DS-SS systems using long PN codes. This assumption has allowed the authors to lump all the $H_{1}$ cells in to a single equivalent $H_{1}$ cell with an appropriate probability of detection; this reduces the analysis of acquisition in multipath to the familiar one with a single $H_{1}$ cell. Owing to the use of short codes and the presence of a large number of multipath components, such an assumption is not justified for the analysis of acquisition of UWB signals. In this paper, we provide a new analysis for the mean acquisition time of conventional serial search without the above assumption.

The fine time resolution of UWB signals and the strict power limitations imposed by the FCC [13] call for innovative ways of acquisition that will result in lower acquisition times. One such acquisition scheme that holds considerable promise is the random search scheme, in which the step size of 
the acquisition receiver is chosen randomly. To the authors' knowledge, acquisition using random search has not been treated analytically in literature, except in [14], where only the 'ideal' case of zero false alarm and unit detection probability is considered. We analyze the acquisition scheme using random search and derive a compact formula for the mean acquisition time for a general scenario.

The rest of the paper is organized as follows. In Section II, the UWB signal model and the channel models under consideration are described. In Section III, we describe the structures and algorithms of the acquisition systems considered in this work. Section IV contains performance analyses of the acquisition systems leading to numerical evaluation of the mean acquisition times and Section V concludes the paper.

\section{SySTEM MODEL}

\section{A. Signal model}

For this work, DS-UWB signaling with bi-phase (BPSK) modulation is assumed. For data transmission, each bit is sent modulated by a length $N_{c}$ PN sequence of \pm 1 binary chips that are periodically repeated in each bit interval, just as in conventional DS-SS systems with short spreading codes. However during the preamble-assisted acquisition phase, it is assumed that an unmodulated PN sequence is transmitted. The UWB signal chosen is a baseband pulse shape given by the $2^{\text {nd }}$ derivative of a Gaussian pulse [14].

$$
p(t)=\sqrt{\frac{4}{3 t_{n} \sqrt{\pi}}}\left(1-\left(\frac{t}{t_{n}}\right)^{2}\right) \exp \left(-\frac{1}{2}\left(\frac{t}{t_{n}}\right)^{2}\right)
$$

The parameter $t_{n}$ determines the effective time width of the pulse $T_{p}$ and hence its bandwidth. The auto-correlation of the pulse $p(t)$ is

$$
R_{p}(\tau)=\left(1-\left(\frac{\tau}{t_{n}}\right)^{2}+\frac{1}{12}\left(\frac{\tau}{t_{n}}\right)^{4}\right) \exp \left(-\frac{1}{4}\left(\frac{\tau}{t_{n}}\right)^{2}\right)
$$

The energy in the pulse $p(t)$ is unity and the signal transmitted with energy $E_{c}$ per chip is thus given by

$$
s(t)=\sqrt{E_{c}} \sum_{k} c_{k} p\left(t-k T_{c}\right)
$$

where $\left\{c_{k}\right\}$ is the pseudo-random code sequence and the chip duration is assumed to be the same as the pulse duration i.e. $T_{c}=T_{p}$.

\section{B. Channel models}

We consider two different wideband channel models - the first is the conventional channel model based on an $L$-tap delay line with a multipath component at each delay and the second is that adopted by the IEEE 802.15.3a task group based on the Saleh-Valenzuela model.

1) Conventional tapped delay-line model: In a timeinvariant multipath channel, the received signal is given by

$$
s_{\text {rec }}(t)=s(t) * h(t)+n(t)
$$

where $h(t)$ is the impulse response of the channel and $n(t)$ is a zero mean additive white Gaussian noise process with a power spectral density of $N_{0}$. The wideband UWB channel can be represented by a tapped delay-line model with the spacing of a minimum multipath resolution $t_{s}$, that is determined by the bandwidth of the system. For convenience, we set $t_{s}=T_{p} / M$, where $T_{p}$ is the pulse duration and $M$ is a positive integer.

$$
h(t)=\sum_{l=1}^{L} \alpha_{l} \delta\left(t-(l-1) t_{s}\right)+n(t)
$$

where $L$ denotes the number of resolvable paths. In our analysis, we assume an exponentially decaying multipath intensity profile with a decay factor of $\eta$; this was assumed as the model for indoor channels in the $2.45 \mathrm{GHz}$ ISM band by the standards group for IEEE 802.11b Wireless LANs. When the total power in all the resolvable paths is normalized to unity, the (average) power in the $l$-th path is expressed as

$$
E\left[\alpha_{l}^{2}\right]=\frac{1-e^{-\eta}}{1-e^{-\eta L}} e^{-(l-1) \eta}
$$

The tap weights are modeled as having independent lognormal distributions, which has been found to be a good fit from some experimental wideband channel measurements [15]. It is important to note that the channel model coefficients are assumed real and not complex; while the complex baseband model is a natural fit for narrowband bandpass systems (to capture the phase dependance as a function of frequency), a real model suffices for a wideband but baseband modulation as in UWB [16].

2) IEEE 802.15.3a channel model: Many indoor channels display a certain 'sparseness' when modeled as a delay line with spacing $1 / B$, i.e. there does not exist a multipath arrival at each resolved lag component. Rather the paths arrive in clusters implying the possibility of a more efficient representation. The model adopted by the IEEE 802.15.3a for UWB propagation in 3-10 $\mathrm{GHz}$ band is a (frequency selective) delay line based on the celebrated Saleh-Valenzuela $(\mathrm{S}-\mathrm{V})$ model [16]. This is characterized by two Poisson distributions for the arrival times - one for the delay of the first path of each cluster $T_{l}$, that has a mean (cluster) arrival rate of $\Lambda$ and the second for the paths within each cluster $\tau_{k, l}$ with a mean (path) arrival rate of $\lambda$. The distributions of the cluster and path arrival times are thus given by

$$
\begin{aligned}
p\left(T_{l} \mid T_{l-1}\right) & =\Lambda \exp \left[-\Lambda\left(T_{l}-T_{l-1}\right)\right] \\
p\left(\tau_{k, l} \mid \tau_{(k-1), l}\right) & =\lambda \exp \left[-\lambda\left(\tau_{k, l}-\tau_{(k-1), l}\right)\right]
\end{aligned}
$$

Therefore, the multipath channel has the following discrete time impulse response.

$$
h(t)=\sum_{l=0}^{L-1} \sum_{k=0}^{K-1} \beta_{k, l} \delta\left(t-T_{l}-\tau_{k, l}\right)
$$

where $\beta_{k, l}$ are the real multipath gain coefficients corresponding to a baseband model. The multipath intensity profile is defined by a double exponential decay model

$$
E\left[\beta_{k, l}^{2}\right]=\Omega_{0} e^{-T_{l} / \Gamma} e^{-\tau_{k, l} / \gamma}
$$

where $\Omega_{0}$ is the mean power of the first path of the first cluster and $\Gamma$ and $\gamma$ are the cluster and path decay factors respectively. The amplitudes are assumed to be independent log-normal distributions. 


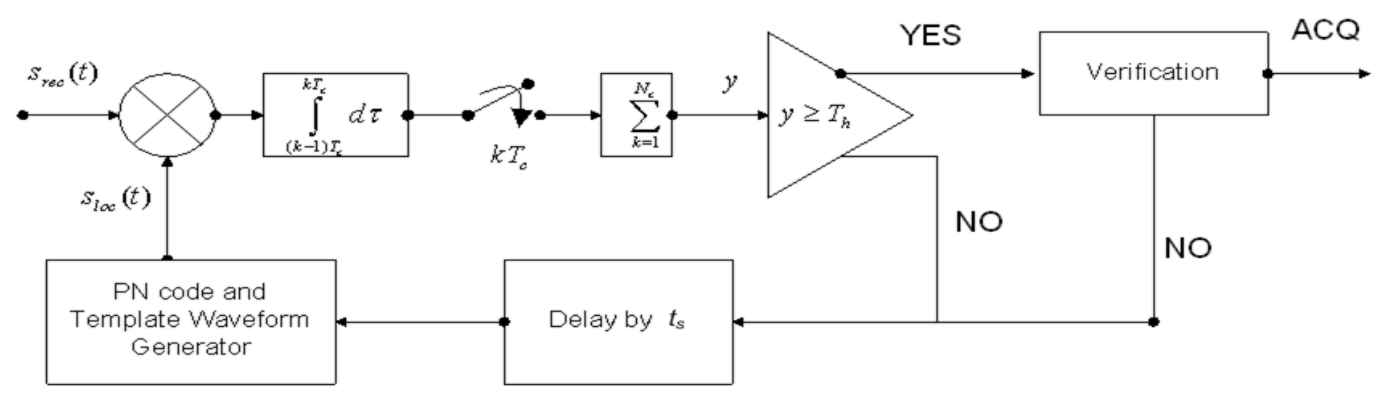

Fig. 1. Structure of an acquisition receiver employing serial search

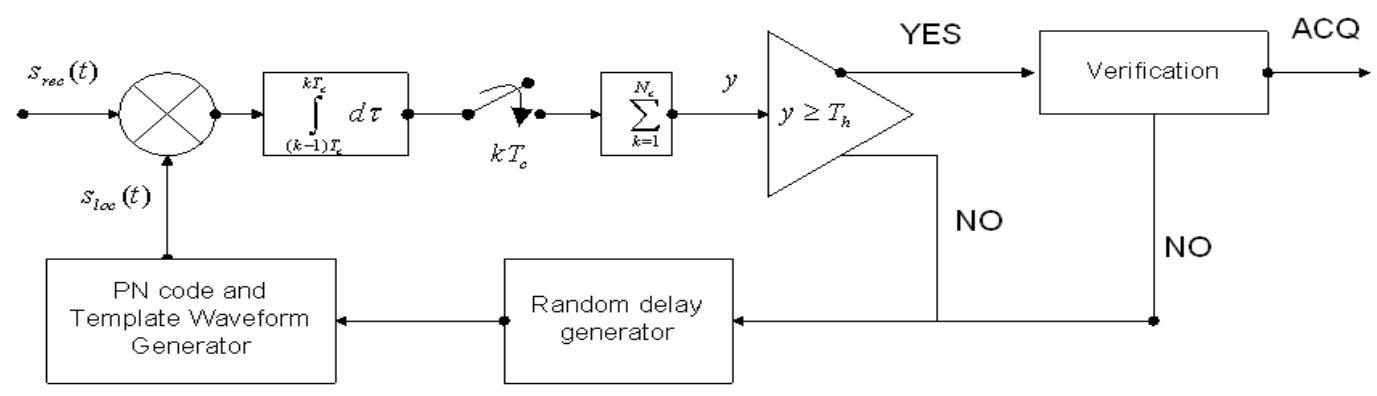

Fig. 2. Structure of an acquisition receiver employing random search

\section{ReCEIVER ARChitecture}

\section{A. Serial Search Acquisition}

The conventional method used to synchronize the received signal with the receiver's local code is the serial search scheme using the sliding correlator [17] shown in Fig 1. The phase of the locally generated code is progressively shifted in sequence in steps of a unit search interval $t_{s}$ (set to be equal to the minimum multipath resolution) and a decision variable formed by correlating the local code with the received code over the correlator dwell time. Since we only consider short codes in this work, the correlator dwell time is assumed to be one full period of the PN code, i.e. $\tau_{D}=N_{c} T_{c}$. The decision variable is then compared with a decision threshold $T_{h}$. If the decision variable exceeds the threshold, the corresponding cell is declared to be an in-phase cell $\left(H_{1}\right.$ cell $)$ and the search is terminated. Otherwise, the cell is declared to be an outof-phase cell $\left(H_{0}\right.$ cell $)$ and the next cell is tested. The entire process is repeated until the codes are aligned (to within a step size ambiguity). The total number of cells in the uncertainty region is thus $q=N_{c} T_{c} / t_{s}=N_{c} M$. In the event of a false alarm, it is assumed that the search resumes after a penalty time of $J$ correlator dwell times, which is the time taken to confirm the false alarm.

\section{B. Random Search Acquisition}

The random search acquisition receiver is very similar to the serial search receiver except that the local code is not shifted serially. Instead, the correlator step size at any time is chosen randomly as shown in Fig 2. The random delay generator changes the phase of the PN code randomly between 1 and $(q-1)$ step sizes. The receiver continues to run with random jumps at each step until acquisition is achieved, which is defined as the state when the receiver locks on to any one of the $H_{1}$ cells to within a step size ambiguity.

\section{Performance Analysis}

We next derive expressions for mean acquisition times (MAT) for the serial and random searches that are verified with simulations. The MAT expressions derived in IV-A.1 and IV-A.2 are new to the best of our knowledge.

\section{A. Performance in conventional multipath model}

Owing to the presence of $L$ multipath components, the uncertainty region in multipath contains $L H_{1}$ cells and the remaining $(q-L)$ cells are $H_{0}$ cells. For an $H_{0}$ cell, the output $y$ of the correlator is a zero-mean Gaussian random variable with a variance of $N_{c} N_{0}$. In the $H_{1}$ cells, the timing error between the local and the received codes is within half a step-size duration. Also, each $H_{1}$ cell in the multipath case is associated with its own probability of detection $P_{D_{l}}$, determined by the corresponding path gain $\alpha_{l}$. Under $H_{1}$ hypothesis for the $l^{\text {th }}$ cell, the decision variable $y$ is now a Gaussian random variable with a variance of $N_{c} N_{0}$, while its mean is equal to $N_{c} \alpha_{l} \sqrt{E_{c}} R_{p}(\xi)$, where $R_{p}(\tau)$ is the normalized pulse auto-correlation function in (2) and $\xi$ is the timing error in a $H_{1}$ cell. We introduce a normalized threshold $\Upsilon_{t h}$ related to $T_{h}$ via $T_{h}=\Upsilon_{t h} N_{c} \sqrt{E_{c}}$. Therefore, for a given fading amplitude and a timing error, the probability of detecting the $l^{\text {th }} H_{1}$ cell is

$$
\begin{aligned}
P_{D_{l}}\left(\Upsilon_{t h} \mid \xi, \alpha_{l}\right)= & \operatorname{Pr}\left(|y|>T_{h} \mid H_{1}\right) \\
= & Q\left(\sqrt{\frac{N_{c} E_{c}}{N_{0}}}\left(\Upsilon_{t h}-\alpha_{l} R_{p}(\xi)\right)\right) \\
& +Q\left(\sqrt{\frac{N_{c} E_{c}}{N_{0}}}\left(\Upsilon_{t h}+\alpha_{l} R_{p}(\xi)\right)\right)
\end{aligned}
$$




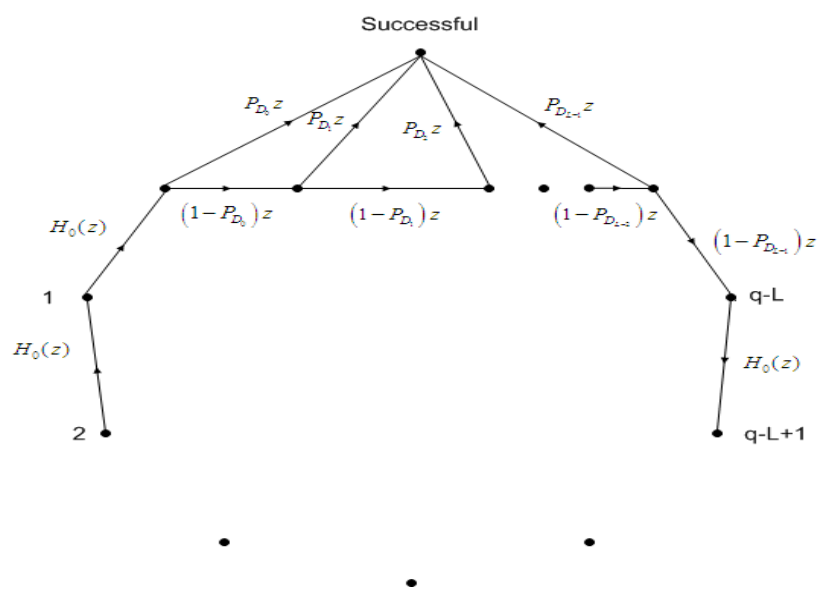

Fig. 3. Circular state diagram for the serial search acquisition system in multipath

It is further assumed that the PN code has perfect autocorrelation properties. Therefore, the mean of $y$ in a $H_{0}$ cell is zero. The probability of false alarm is then given by

$$
P_{F A}\left(\Upsilon_{t h}\right)=\operatorname{Pr}\left(|y|>T_{h} \mid H_{0}\right)=2 Q\left(\sqrt{\frac{N_{c} E_{c}}{N_{0}}} \Upsilon_{t h}\right)
$$

1) Serial Search: The traditional analyses of the serial search scheme performed in [11] and [12] make the assumption that the number of $H_{1}$ cells $(L)$ is negligible compared to the total number of cells $(q)$ in the search space; this is clearly invalid for the acquisition of UWB signals. In the following, we analyze the serial search scheme without the assumption. In order to do this, we consider the transfer function starting from a $H_{0}$ cell to the $A C Q$ state as distinct from that starting from a $H_{1}$ cell to the $A C Q$ state. The relevant state diagram for acquisition in multipath is shown in Fig 3.

(i) Given that the search starts in a $H_{0}$ cell that is $i$ cells to the left of the first $H_{1}$ cell, the transfer function to reach the ACQ state is given by

$$
H_{i}(z)_{\left(H_{0}\right)}=\frac{H_{0}^{i}(z) H_{D}(z)}{1-H_{M}(z) H_{0}^{(q-L)}(z)}
$$

where $H_{0}(z)$ is the transfer function for exiting a $H_{0}$ cell, $H_{D}(z)$ is the transfer function denoting absorption into the ACQ state starting from the first $H_{1}$ cell before encountering a $H_{0}$ cell and $H_{M}(z)$ is the transfer function denoting a total miss in any round.

$$
\begin{aligned}
H_{0}(z) & =P_{F A} z^{J+1}+\left(1-P_{F A}\right) z \\
H_{D}(z) & =\sum_{j=1}^{L} P_{D_{j}} z \prod_{k=1}^{j-1}\left(1-P_{D_{k}}\right) z \\
H_{M}(z) & =\prod_{j=1}^{L}\left(1-P_{D_{j}}\right) z
\end{aligned}
$$

(ii) Given that the initial cell is the $i^{\text {th }} H_{1}$ cell, the transfer function to reach the ACQ state is given by

$$
H_{i}(z)_{\left(H_{1}\right)}=H_{D_{r}}(z)+H_{M_{r}}(z) H_{q-L}(z)_{\left(H_{0}\right)}
$$

where $H_{D_{r}}(z)$ is the transfer function denoting absorption into the ACQ state starting from the $i^{\text {th }} H_{1}$ cell before encountering a $H_{0}$ cell and $H_{M_{r}}(z)$ is the transfer function of missing the $H_{1}$ cells numbered $i$ through $L$.

$$
\begin{aligned}
H_{D_{r}}(z) & =\sum_{j=i}^{L} P_{D_{j}} z \prod_{k=i}^{j-1}\left(1-P_{D_{k}}\right) z \\
H_{M_{r}}(z) & =\prod_{j=i}^{L}\left(1-P_{D_{j}}\right) z
\end{aligned}
$$

Since each cell has a uniform probability of $1 / q$ of being the starting cell, the overall transfer function of the serial search is given by

$$
H(z)=\frac{1}{q}\left[\sum_{i=1}^{q-L} H_{i}(z)_{\left(H_{0}\right)}+\sum_{i=1}^{L} H_{i}(z)_{\left(H_{1}\right)}\right]
$$

The mean acquisition time can then be obtained by

$$
\bar{T}_{a c q}=\left.\frac{d H(z)}{d z}\right|_{z=1} \times \tau_{D}
$$

$\bar{T}_{a c q}$ is given by (16) at the bottom of this page, where

$$
\begin{aligned}
T_{D} & =\sum_{j=1}^{L} j P_{D_{j}} \prod_{k=1}^{j-1}\left(1-P_{D_{k}}\right) \\
P_{M} & =\prod_{k=1}^{j-1}\left(1-P_{D_{k}}\right)
\end{aligned}
$$

At high signal-to-noise ratios when $P_{F A} \rightarrow 0$ and $P_{D_{i}} \rightarrow$ $1, \forall i$, the process takes one integration time to reach the ACQ state if the search falls in any of the $H_{1}$ cells and $(i+1)$ integration times if it falls in a $H_{0}$ cell $i$ cells to the left of the first $H_{1}$ cell. Therefore, $\bar{T}_{a c q}$ reduces to

$$
\begin{aligned}
\bar{T}_{a c q}(\text { sat }) & =\frac{1}{q}\left[L+\sum_{i=1}^{q-L}(i+1)\right] \times \tau_{D} \\
& =\frac{2(L-1)+(q-L+1)(q-L+2)}{2 q} \tau_{D}
\end{aligned}
$$

$$
\begin{aligned}
\bar{T}_{a c q}= & \frac{q-L}{q}\left(\frac{2 T_{D}+\left(q+q P_{M}-L+1-P_{M}\right)\left(1+J P_{f}\right)+L P_{M}\left(1-J P_{f}\right)}{2\left(1-P_{M}\right)}\right) \tau_{D} \\
+ & \frac{1}{q}\left(\sum_{i=1}^{L} \sum_{j=i}^{L}(j-i+1) P_{D_{j}} \prod_{k=i}^{j-1}\left(1-P_{D_{k}}\right)\right) \tau_{D} \\
& +\frac{1}{q}\left(\sum_{i=1}^{L}\left(\frac{T_{D}+(q-L)\left(1+P_{f} J\right)+L P_{M}}{\left(1-P_{M}\right)}+(L-i+1)\right)\left(\prod_{k=i}^{L}\left(1-P_{D_{j}}\right)\right)\right) \tau_{D}
\end{aligned}
$$




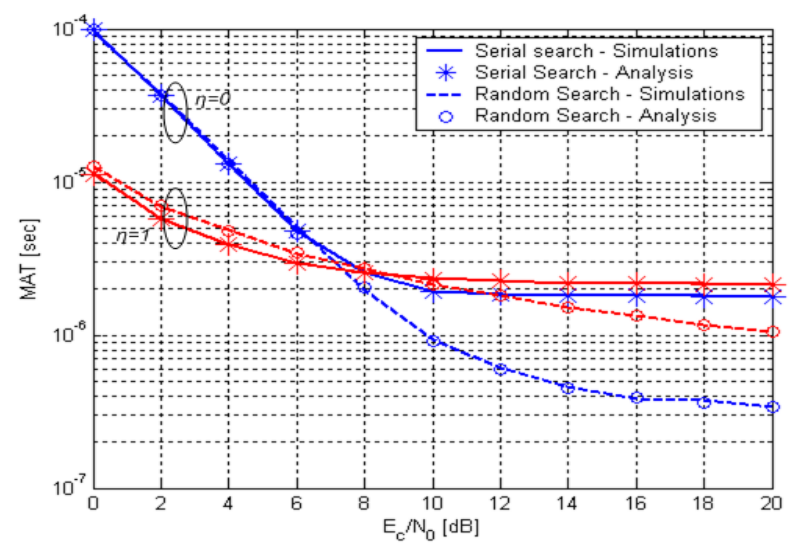

Fig. 4. Mean acquisition time in conventional multipath channel: $\mathrm{L}=16$

2) Random Search: The state diagram of random search would have the $H_{1}$ cells dispersed randomly among the $H_{0}$ cells. The overall transfer function can still be derived by noting that when the process is in a $H_{0}$ cell, it has a $1-L / q$ probability of transitioning to a $H_{0}$ cell in the next step and a $1 / q$ probability of transitioning to each of the $H_{1}$ cells. Therefore, the transfer function for exiting an $H_{0}$ cell is given by

$$
\begin{aligned}
H_{0}(z)= & {\left[P_{F A} z^{J+1}+\left(1-P_{F A}\right) z\right] \times } \\
& {\left[\left(\frac{q-L}{q}\right) H_{0}(z)+\left(\frac{1}{q}\right) \sum_{i=1}^{L} H_{i}(z)\right] }
\end{aligned}
$$

where $H_{i}(z)$ is the transfer function for exiting the $i^{\text {th }} H_{1}$ cell. On the other hand, when the search is in the $i^{t h} H_{1}$ cell, it could move to the $A C Q$ state with a probability of $P_{D_{i}}$, to a $H_{0}$ cell with a probability of $\left(1-P_{D_{i}}\right)(q-L) / q$ and to each of the remaining $H_{1}$ cells with a probability of $\left(1-P_{D_{i}}\right) 1 / q$. Therefore the transfer function from the $i^{t h} H_{1}$ cell is given by

$$
\begin{aligned}
H_{i}(z)= & P_{D_{i}} z+\left(1-P_{D_{i}}\right) z \times \\
& {\left[\left(\frac{q-L}{q}\right) H_{0}(z)+\left(\frac{1}{q}\right) \sum_{j=1}^{L} H_{j}(z)\right] }
\end{aligned}
$$

The $H_{i}(z)$ 's and $H_{0}(z)$ can be obtained by simultaneously solving the $L+1$ equations obtained from (19) and (20). Since the search has a $(q-L) / q$ probability of starting in a $H_{0}$ cell and a $1 / q$ probability of starting in each of the $H_{1}$ cells, the effective transfer function to reach the $A C Q$ state is given by

$$
H(z)=\left(\frac{q-L}{q}\right) H_{0}(z)+\left(\frac{1}{q}\right) \sum_{i=1}^{L} H_{i}(z)
$$

The mean acquisition time is again calculated using (15). For random search, the formula for the mean acquisition can be shown to be given by the closed form

$$
\bar{T}_{a c q}=\frac{P_{F A} J(q-L)+q}{\sum_{l=1}^{L} P_{D_{l}}} \times \tau_{D}
$$

In random search at high signal-to-noise ratio values, the probability that the search lands in a $H_{1}$ cell is $L / q$ and the

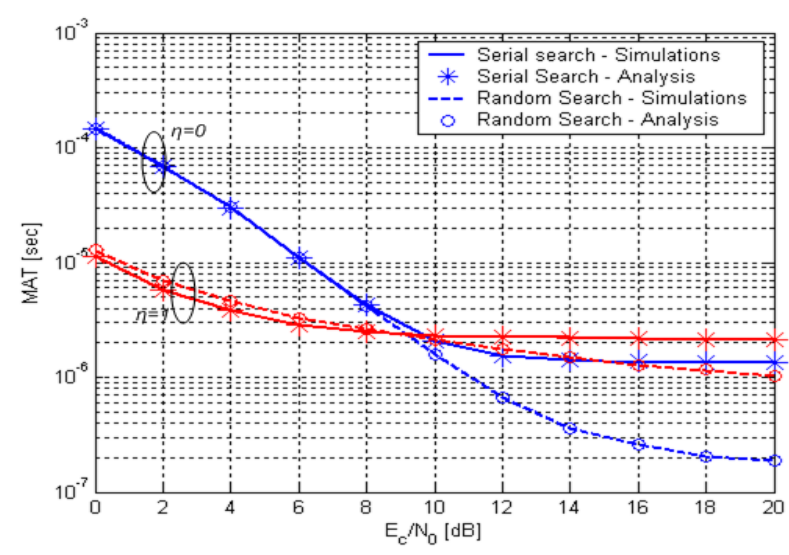

Fig. 5. Mean acquisition time in conventional multipath channel: $\mathrm{L}=32$

number of jumps to get to a $H_{1}$ cell follows a geometric distribution with a mean of $q / L$. Therefore the mean acquisition time at high signal-to-noise ratios is

$$
\bar{T}_{a c q}(s a t)=\left(\frac{q}{L}\right) \tau_{D}
$$

which is the same as predicted by the above formula.

3) Simulations: We verified the above observations by means of simulations using a UWB pulse of width $1 \mathrm{~ns}$ and a PN code of length 32 . The multipath channel having an exponential power delay profile was simulated with different delay spreads and decay factors. The threshold setting was based on constant false alarm rate (CFAR) with $P_{F A}=10^{-4}$ and the step size for the correlator was fixed to be a fourth of the pulse duration, i.e., $M=4$. Fig 4 shows the results of the simulations for a delay spread of 4 ns and Fig 5 for a delay spread of $8 \mathrm{~ns}$. Both the figures show results for two decay factors, $\eta=0$ (uniform MIP) and $\eta=1$.

The first observation is that the simulation results for the serial and random searches match exactly those predicted by analysis. Also it is clear that it becomes increasingly advantageous to adopt the random search over serial search as the multipath profile becomes more uniform. The advantage of random search is that it requires fewer steps on the average to reach a $H_{1}$ cell than serial search when the number of paths is large. On the other hand, the serial search has the advantage that it encounters cells with higher probability of detection earlier than those with lower probability of detection when the multipath intensity profile is exponential (not so when the profile is more uniform). It thus takes a larger SNR for the natural advantage of random search to outweigh that of serial search for an exponential multipath profile as the decay factor is increased.

The performance advantage of random search over serial search as a function of the number of multipath components is examined by comparing Figs 4 and 5. It is evident that the advantage increases as the number of multipaths increases. This is due to the fact that as the number of paths increases, the mean number of steps required to reach a $H_{1}$ cell decreases faster for random search than for serial search. In summary, 


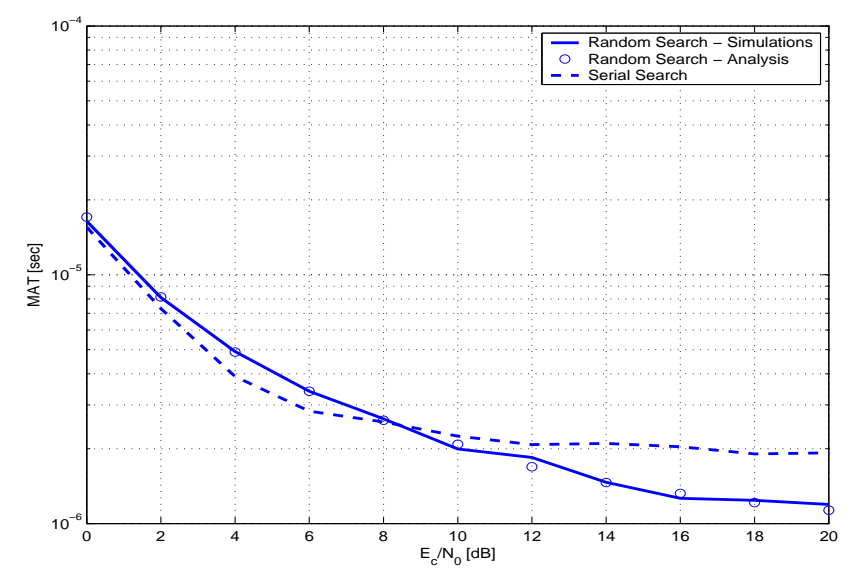

Fig. 6. Mean acquisition time in the IEEE 802.15.3a channel model

random search gives the most benefit when the multipath profile is uniform and the number of multipath components is large.

\section{B. Performance in IEEE 802.15.3a channel model}

The analysis of the random search scheme performed in Section IV-A.2 and the expression for mean acquisition time derived therein do not make any assumption about the channel model. Since the channel model only affects the probabilities of detection and false alarm, the MAT formula in (22) can be used for the IEEE802.15.3a channel model as well. The serial search analysis in Section IV-A.1 however is based on the assumption that multipath components are present on contiguous tap positions and therefore would not apply for the IEEE 802.15.3a model. The fact that there is a finite probability that there could be $H_{0}$ cells interspersed among the $H_{1}$ cells complicates the analysis. We therefore resorted to simulations to evaluate the performance of the serial search scheme.

For our simulations, we still considered a UWB pulse of width $1 \mathrm{~ns}$ and a PN sequence of length 32 . We simulated a channel based on the IEEE 802.15.3a model with $1 / \Lambda=10 \mathrm{~ns}$ and $1 / \lambda=1 \mathrm{~ns}$. Threshold setting was based on CFAR with a $P_{F A}=10^{-4}$. The results of the simulations are plotted in Fig 6 , which shows the acquisition times of the conventional serial search and random search schemes. For random search, the MAT values obtained from the formula match those obtained from simulations pretty well. Also noticeable is the fact that the performance of serial search is not significantly different from random search. The inherent randomness of the multipath arrivals in the 802.15.3a channel model renders serial search performance to be very similar to random search. As the multipath arrivals become more random, i.e., the $H_{1}$ cells become more uniformly dispersed among $H_{0}$ cells, the performance of serial search scheme would become indistinguishable from that of random search. This underscores the broad utility of the random search analysis developed - it can be used as a good model for any deterministic search scheme where the multipath profile is 'randomized'.

\section{CONCLUSIONS}

We provide a new analysis of serial search by removing assumptions invoked for conventional wideband systems that are not valid for UWB. We have also derived an expression for the mean acquisition time for random search for any general multipath channel model. It has been shown that the random search provides significant improvement in performances over serial search when the number of multipath components is large and especially when the multipath profile is more uniform. Typical UWB channel models tend to have random multipath arrivals, and any deterministic search scheme would behave much like random search. Thus the analysis provided in this paper should prove to be very useful in estimating the acquisition times in typical UWB channels.

\section{REFERENCES}

[1] M. Z. Win and R. A. Scholtz, "Impulse radio: How it works," in IEEE Communication Letters, vol. 2, pp. 36-38, Feb 1998.

[2] K. Siwiak, "Ultra-wideband radio: Introducing a new technology," in Proc. 2001 Spring Vehicular Technology Conf., pp. 1088-1093, 2001.

[3] S. Roy, J. R. Foerster, V. Somayazulu, and D. G. Leeper, "Ultrawideband radio design: The promise of high-speed, short-range wireless connectivity," in Proc. of the IEEE. Special Issue on Gigabit Wireless, vol. 92, no. 2, pp. 292-311, Feb 2004.

[4] M. Z. Win and R. A. Scholtz, "On the robustness of ultra-wide bandwidth signals in dense multipath environments," in IEEE Communication Letters, vol. 2, pp. 51-53, Feb 1998.

[5] M. Z. Win and R. A. Scholtz, "Ultra-wide bandwidth time-hopping spread-spectrum impulse radio for wireless multiple-access communications," in IEEE Transactions on Communications, vol. 48, no. 4, pp. 679-691, Apr 2000.

[6] J. R. Foerster, "The performance of a direct-sequence spread ultrawideband system in the presence of multipath, narrowband interference, and multiuser interference," in IEEE Conference on Ultra Wideband Systems and Technologies (UWBST), pp. 87-91, May 2002.

[7] A. Batra et al., "Multi-band ofdm physical layer proposal for ieee 802.15 task group 3a." http://www.multibandofdm.org/papers/ 15-03-0268-01-003a-Multi-band-CFP-Document.pdf.

[8] R. "Xtremespectrum CFP document." http://grouper.ieee.org/groups/ 802/15/pub/2003/Jul03/03154r3P802-15_ TG3a-XtremeSpectrum-CFP-Documentation.pdf.

[9] A. Polydoros and C. Weber, "A unified approach to serial search spread-spectrum code acquisition-Part I: General theory," in IEEE Trans Commun, vol. 32, no. 5, pp. 542-549, May 1984.

[10] J. K. Holmes and C. C. Chen, "Acquisition time performance of PN spread-spectrum systems," in IEEE Trans Commun, vol. 25, no. 8, pp. 778-784, Aug 1977.

[11] L. L. Yang and L. Hanzo, "Serial acquisition of DS-CDMA signals in multipath fading mobile channels," in IEEE Trans. Veh. Technol., vol. 50, no. 2, pp. 617-628, Mar 2001.

[12] B. B. Ibrahim and A. H. Aghvami, "Direct sequence spread spectrum matched filter acquisition in frequency-selective Rayleigh fading channels," in IEEE J. Select. Areas Commun., vol. 12, pp. 885-890, June 1994.

[13] Federal Communications Commission, "Revision of part 15 of the commissions rules regarding ultra-wideband transmission systems: First report and order," tech. rep., FCC, Apr. 2002.

[14] E. A. Homier and R. A. Scholtz, "Rapid acquisition of ultra-wideband signals in the dense multipath channel," in IEEE Conference on Ultra Wideband Systems and Technologies (UWBST), pp. 105-109, May 2002.

[15] J. R. Foester et. al., "Channel modeling sub-committee report final,", tech. rep., IEEE P802.15 Wireless Personal Area Networks, P802.1502/490r1-SG3a, Feb. 2003.

[16] A. F. Molisch, J. R. Foerster, and M. Pendergrass, "Channel models for ultrawideband personal area networks," in IEEE Wireless Communications, pp. 14-21, Dec 2003.

[17] A. J. Viterbi, CDMA: Principles of spread spectrum communication. MA, U. S. A: Addison-Wesley, 1995. 